\title{
INTERPOLATION OF MAGNETIC AND ELECTRIC FIELDS USING SPHERICAL ELEMENTARY CURRENT SYSTEMS
}

\author{
C.D. Beggan*, S.A. McLay ${ }^{\dagger}$ \\ *British Geological Survey, UK (Murchison House, West Mains Road, Edinburgh, EH9 3LA; ciar@bgs.ac.uk) \\ ${ }^{\dagger}$ Chevron Upstream Europe, Chevron North Sea Limited, Seafield House, Hill of Rubislaw, Aberdeen, AB15 6XL
}

Keywords: Magnetic field; Electric field; Interpolation; Extrapolation; Earth Hazards

\begin{abstract}
A physics-based technique for interpolating magnetic and electric field disturbances of external origin across large spatial areas can be achieved by employing the Spherical Elementary Current System (SECS) method using data from ground-based magnetic observatories. The SECS method represents complex electrical current systems as a simple set of equivalent currents placed at a specific height in the ionosphere. The magnetic field recorded at observatories can be used to invert for the electrical currents and subsequently employed to interpolate or extrapolate the electric and magnetic field across a large area at mid- to high geomagnetic latitudes. Here we show that the magnetic field interpolation can be improved, even over very large distances (> $1000 \mathrm{~km})$, by the addition of further observatory data into the SECS inversion.
\end{abstract}

\section{Introduction}

On time periods of seconds to days, changes in the magnetic field are principally of external origin and are caused by the interaction of the solar wind, magnetosphere and ionosphere/upper atmosphere system. The study of temporal changes in the geomagnetic field and the accompanying electric fields are of significant interest in Earth hazard research.

There are two classes of technology adversely affected by space weather: those directly affected by variations in the geomagnetic field (such as navigation systems) and those affected by the electric currents induced by the changing field (such as buried pipelines). Modern applications of space weather studies to ground-based technology include the problems associated with Geomagnetically Induced Currents within power grids, oil and gas pipelines, telecommunication cables and railway equipment [7]. Indirectly, geomagnetic disturbances in the ionosphere/upper atmosphere can disrupt the operation of technology exploiting real-time satellite data, such as positioning and navigation systems. For these reasons, during geomagnetic storms or extreme events, it is useful to have an estimate of the magnetic and electric field to monitor the impact at a specific position.
However, reliable interpolation or extrapolation of the external magnetic field as observed at the surface of the Earth across a large distance is difficult; in part due to large and complex spatial variations that occur in ionospheric current systems. Several techniques have been developed to calculate equivalent currents from ground-based measurements; two of the most successful thus far have been the Fourier method [6] and Spherical Cap Harmonic Analysis (SCHA) [4]. A third approach, the Spherical Elementary Current System method, was established and developed by [1] and [2]. The use of SECS overcomes some of the limitations of the other two methods and is suitable for studies on small and large regional scales. The SECS technique was demonstrated to produce a better fit than SCHA to simulated ionosphere conditions in [1], while [8] showed it is applicable for the interpolation of ionospheric fields in a densely sampled, relatively small region around northern Scandinavia.

The use of SECS to robustly extrapolate the electrical field over a large region in North America was demonstrated in [5] and [9], by applying the SECS technique to a large nonrectangular area with widely-spaced ground magnetometers $(<350 \mathrm{~km})$. In this paper we present new results using the THEMIS ground variometer network [3] to test the ability of the SECS method to improve the interpolation of the electric and hence magnetic field over even larger regions $(>1000$ $\mathrm{km})$.

\section{Spherical Elementary Current Systems}

The implementation of the SECS method, which we briefly summarise here, is outlined in detail in [5] for example. The basic concept of SECS is to construct a set of equivalent current systems using a linear superposition of divergencefree elementary current systems, all of which can be placed freely within one or two current planes [1]. The method is derived from Maxwell's equations giving a physical rather than a mathematical approach to interpolation.

Within this description, we define of current planes for (a) the ionosphere $\left(\mathrm{R}_{\mathrm{s}}\right)$ and $(\mathrm{b})$ the subsurface $\left(\mathrm{R}_{\mathrm{e}}\right)$. For example, a single elementary current system for the external field, defined at a pole, is described by:

$$
\mathbf{J}_{\mathbf{d f}}(\mathrm{r}, \theta)=\mathrm{I}^{\mathrm{e}} / 4 \pi \mathrm{R}_{\mathrm{S}} \cot (\theta / 2)
$$


where $\mathrm{I}^{\mathrm{e}}$ are the scaling factors for the external divergencefree current systems and $R_{S}$ is the radius of the ionosphere. The magnetic field vector (B) at the surface of the Earth at a location $(r, \theta, \varphi)$ can then be computed by the superposition of the magnetic effect of the external and internal layers of horizontal currents consisting of a series of elementary current systems:

$$
\begin{aligned}
\mathbf{B}(\mathrm{r}, \theta, \varphi) & =\sum_{j=1}^{L} I_{j}^{i} T_{d f}^{i}\left(\mathrm{R}_{\mathrm{G}}, \theta_{j}, \varphi_{j}, r, \theta, \varphi\right) \\
& +\sum_{k=1}^{M} I_{k}^{e} T_{d f}^{e}\left(\mathrm{R}_{\mathrm{S}}, \theta_{k}, \varphi_{k}, r, \theta, \varphi\right)
\end{aligned}
$$

where $T_{d f}^{e}$ (derived from Equation (1)) and $T_{d f}^{i}$ are the geometric parts of the external and internal relating the current systems to magnetic fields produced by each elementary current system and $L$ and $M$, with subscripts $j$ and $k$ denote the number of current systems solved, specified at the radius of the ionosphere and at depth within the subsurface (typically $100 \mathrm{~km}$ ), respectively.

\subsection{Inversion for the current systems}

We assume that the magnetic field vector $\mathbf{B}$ has been measured at a set of points and construct a linear system of equations relating the measured field to the geometric parts and scaling factors of both the internal and external elementary current systems. Expressing this in matrix form gives:

$$
\mathbf{B}=\mathbf{T} \cdot \mathbf{I}
$$

We look to solve the linear inverse problem to determine the scaling factors $\mathbf{I}$ of the internal and external elementary current systems. Due to the (generally) limited number of fixed ground magnetic observatories, the number of observation points is usually much lower than the number of elementary current systems required to produce a good representation of the actual currents. The linear inverse problem is therefore highly-underdetermined:

$$
\mathbf{I}=\mathbf{T}^{-\mathbf{1}} \cdot \mathbf{B}
$$

The matrix $\mathbf{T}$ may be badly conditioned which produces numerical instabilities when attempting to invert Equation (4) directly. Thus, we employ Singular Value Decomposition for the inversion, truncating such that any eigenvalues with an absolute value less than 1/100th of the largest are excluded from the solution. The truncation stabilises the inversion and tends to produce a small smoothing effect on the solution. Other truncation levels were tested, including the truncation of eigenvalues with an absolute value less than 1/10th of the largest value and also zero truncation. The results were stable and generally similar; suggesting in these experiments that the $\mathbf{T}$ matrix is not actually that badly conditioned.

Modelling was undertaken using minute-mean magnetic field vector values. To prepare the data for inversion, the main (core) and local (crustal) fields for each day were removed by subtracting the mean daily baseline values from the North (X), East (Y) and Downward (Z) components, giving a 24-hour set of magnetic disturbance values around a daily average. This deviation from the mean was used as the B matrix and the geographic positions of the observatories were used to construct the $\mathbf{T}$ matrix. The scaling factors (I) for the current systems across the grid of points were solved for every minute of the day.

We solve for the scaling factors of the currents systems on a rectangular grid evenly-spaced in latitude and longitude (though, any reasonable shape and spacing could be used). Thus, the forward solution for the magnetic field $(\mathbf{B})$ on the Earth's surface at any position within the grid can be calculated by determining the $\mathbf{T}$ matrix for the point of interest and using the scaling factors from the inversion.

\subsection{Estimation of interpolation accuracy}

The accuracy of the interpolation method can be quantified by comparing the estimated magnetic field to magnetic field data measured at an observatory which has not been used in the inversion. The root-mean-square (RMS) difference between the estimated magnetic field and the measured field at a particular location averaged over one day of minute-mean data for a set of $n$ points can be calculated as:

$$
\mathrm{RMS}_{\text {error }}=\sqrt{\Sigma}\left[\left(\mathrm{B}_{\text {obs }-} \mathrm{B}_{\mathrm{SECS}}\right)^{2} / n\right]
$$

where $B_{\text {obs }}$ is the measured field at an observatory at each minute during a day and $\mathrm{B}_{\mathrm{SECS}}$ is the estimated field, for each of the three components of the magnetic field vector. The power of the measured data is described by the root-sumsquare of the values, giving a measure of the magnetic disturbance for a given day of data:

$$
\text { Power }=\sqrt{ } \Sigma\left(\mathrm{B}_{\mathrm{obs}}\right)^{2} / n
$$

The study of [5] showed that at high geomagnetic latitudes, the SECS method gave the best estimate of the external magnetic field, particularly during magnetically active days, when compared to other mathematical interpolation methods such as the latitudinal-weighted average value of two nearby observatories. Here we examine the effects of additional observatories or variometer data within the SECS inversion.

\section{Applying SECS to Magnetic Field Data}

The magnetic field as measured at the Yellowknife magnetic observatory (observatory code: YKC) in the central Canadian Shield is used as a test point throughout this study. Data from variometers associated with the ground segment of the THEMIS mission were retrieved from the University of Berkeley. As most of the variometer stations were set up from 2007 onwards, 110 days of minute-mean data for the period of February to May 2007 were selected. Eight variometers were found to have sufficiently continuous data coverage for use in this study. Minute mean data covering the same time 


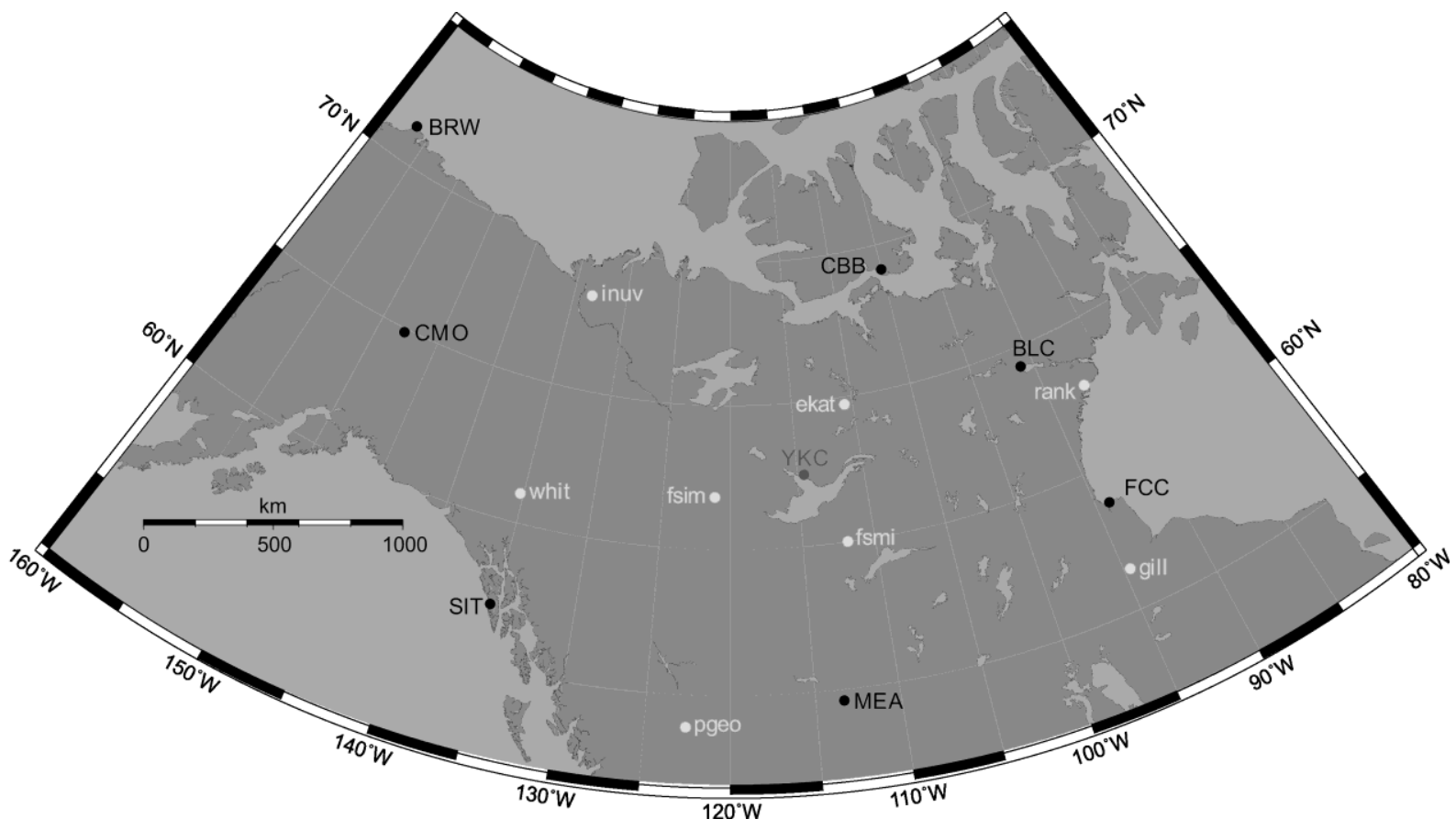

Figure 1: Locations of the three observatories operated by the United States Geological Survey [BRW; CMO; SIT] and four observatories operated by the Geological Survey of Canada [BLC; CBB; FCC; MEA] (black) and the eight variometers from the THEMIS ground network (light grey). The position of Yellowknife observatory operated by the Geological Survey of Canada $[\mathrm{YKC}]$ at the centre is also shown.

period for YKC and seven other permanent magnetic observatories operating in the region were obtained from the World Data Centre for Geomagnetism in Edinburgh in 2010.

Figure 1 shows the location of the observatories and variometers about the Yellowknife observatory. Three

variometers (ekat, $f_{s i m}, f_{s m i}$ ) are sited close the YKC observatory. The remaining five (gill, inuv, pgeo, rank, whit) are at larger distances from $\mathrm{YKC}$ comparable to the observatories used (BLC, BRW, CBB, CMO, FCC, MEA, SIT).

The long term comparison (approximately four months) of the estimate for YKC was computed using the following sets of observatories and variometers:

Case 1: Seven observatories only

Case 2: Seven observatories and three close variometers

Case 3: Seven observatories and five distant variometers

Case 4: Seven observatories and all eight variometers

A rectangular grid of current systems evenly-spaced in latitude and longitude was constructed with a grid spacing of $2^{\circ}$. The estimates of the magnetic field have been shown to be relatively insensitive to varying the grid spacing between 0.5 and $2.0^{\circ}$ [5]. The scaling factors (I) for the current systems across the grid of points were solved for every minute of every day. The magnetic field at YKC was then estimated and the RMS difference between the recorded data and the interpolated value at the observatory for each day was computed.

\section{Results}

The results of the RMS comparison (Equation (5)) of 110 days of minute-mean data are shown in Figure 2. Table 1 gives the mean of the differences across the 110 days, excluding days without data. The comparison with YKC of the SECS estimate using the seven observatories (Case 1, black solid) shows that only the X component of the SECS estimate is better than the assumption of no field change (i.e. the power from Equation (6), grey dashed). The figures in Table 1 confirm this - the power in $\mathrm{X}$ is $59.1 \mathrm{nT}$ while the difference of the SECS estimate is $41.2 \mathrm{nT}$. The $\mathrm{Y}$ and $\mathrm{Z}$ components are poorly estimated in this situation and are in fact worse in general than estimating that no change occurred in the magnetic field (i.e. the black solid line lies above the grey dashed). The reason for the poor performance is likely to be due to the distance between the observatories as this is the largest network tested at about $4 \times 10^{6} \mathrm{~km}^{2}$. This could be considered as a worst-case scenario as we are interpolating using the SECS methodology over large distances to a point (test observatory) lying within the auroral zone where the external field is prone to its largest variations.

The local variometers should experience similar external field conditions to YKC. Using data from the variometer stations close to the point of interest strongly improves the estimate (Case 2, grey dotted), particularly in the $\mathrm{X}$ and $\mathrm{Z}$ component. Table 1 shows the mean RMS differences are 23.5 and 25.5 nT, respectively. The SECS estimate using data from the five most distant variometers and the observatories produces an interesting result (Case 3, black dash). Using the five 
variometers at distances similar to the seven observatories shows a marked improvement in all three components, particularly in X and Y (mean RMS differences are now 34.9 and $27.2 \mathrm{nT}$ ), though the $\mathrm{Z}$ component is still poorly estimated on noisy days or days with some missing data (e.g. Days 53 and 73). In the $\mathrm{Z}$ component, there is little improvement when compared with the SECS estimate from the observatory data alone. This suggests that it is very difficult to estimate the field induced locally in the crust using only data from distant observations. However, the results do suggest that adding data from stations which are distant can improve the estimate in the $\mathrm{X}$ and the $\mathrm{Y}$ component, which is a useful result.

Using all seven observatories and eight variometers produces the best estimate overall (Case 4, grey solid), in X and Y, and quite often in $\mathrm{Z}$ also. This again suggests that distant observatories have a positive influence on the estimate of the field. The improvement in $\mathrm{Z}$ is most likely due to the better estimate of the internal induced field local to the site, a product of using the three closest variometers.

On average, according to Table 1, using all data available does reduce the difference on most days, suggesting that it is advantageous to use as many data as possible when computing the interpolation. It is also clear that the closer variometers/magnetometers help to reduce the misfit in the vertical component of the interpolated magnetic field (compare the grey dotted and grey solid lines is Figure 2).

\section{Conclusions}

In almost all cases the SECS method proves to be better than assuming no change of the magnetic field and thus it is worthwhile using the technique to correct for external field disturbances even during magnetically quiet conditions or to estimate the electrical field strength within the ionosphere.

The most interesting result shows the addition of more stations, even distant ones, improves the estimate of the magnetic and electric field in the $\mathrm{X}$ and $\mathrm{Y}$ components. This suggests that it is possible to produce a good estimate in a central region surrounded by observatories/variometers. This is applicable to a number of regions in the world, where it is not possible to place suitable observing equipment (e.g. offshore). In addition, it has been shown that local variometer data are essential for the accurate prediction of the $\mathrm{Z}$ component of the magnetic field where locally induced currents have a strong influence.

The SECS methodology provides a more sophisticated external field interpolation and is now being applied, for example, at the British Geological Survey to improve the prediction of Geomagnetically Induced Currents in UK electrical power distribution network during geomagnetic storm events.

We have shown that the SECS method can be applied networks of different sizes where various densities and geometries of observatory data are available. Additional observatory data does improve the estimate from SECS, particularly when close to the region of interest. We conclude that the SECS method can be used effectively to improve the magnetic and electric field estimate at a point remote from an observatory, which will be useful in ionospheric and external field studies.

\section{Acknowledgements}

This research was partly funded by NERC New Investigators grant NE/J004693/1. The institutes that support magnetic observatories together with INTERMAGNET are thanked for promoting high standards of observatory practice. Observatory data were obtained from the World Data Centre, Edinburgh (www.wdc.bgs.ac.uk). Variometer data was acquired from University of Berkeley in 2010 (themis.ssl.berkeley.edu). This article is published with the permission of the Director of the British Geological Survey (NERC).

\section{References}

[1] O. Amm. "Ionospheric elementary current systems in spherical coordinates and their application", Journal of Geomagnetism and Geoelectricity, 49, pp. 947-955, (1997).

[2] O. Amm, and A. Viljanen. "Ionospheric disturbance magnetic field continuation from the ground to the ionosphere using spherical elementary current systems", Earth Planets Space, 51, pp. 431-440, (1999).

[3] V. Angelopoulos. "The THEMIS mission", Space Science Reviews, 141, pp. 5-34, (2008).

[4] G. Haines. "Spherical cap harmonic analysis", Journal of Geophysical Research, 90, pp. 2583-2591, (1985).

[5] S. A. McLay and C. D. Beggan. "Interpolation of externally-caused magnetic fields over large sparse arrays using spherical elementary current systems", Annales Geophysicae, 28, pp. 1795-1805, (2010).

[6] U. Mersmann , W. Baumjohann, F. Küppers, and K. Lange. "Analysis of an eastward electrojet by means of upward continuation of ground-based magnetometer data", Journal of Geophysics, 45, pp. 281-298, (1979).

[7] R. Pirjola, A. Viljanen, A. Pulkkinen, and O. Amm. "Space weather risk in power systems and pipelines", Physics and Chemistry of the Earth, 25, pp. 333 -337, (2000).

[8] A. Pulkkinen, O. Amm, A. Viljanen, and BEAR Working Group. "Ionospheric equivalent current distributions determined with the method of spherical elementary current systems", Journal of Geophysical Research, 108(A2), pp. 1053, (2003).

[9] J. M. Weygand, O. Amm, A. Viljanen, V. Angelopoulos, D. Murr, M. J. Engebretson, H. Gleisner, and I. Mann. "Application and validation of the spherical elementary currents systems technique for deriving ionospheric equivalent currents with the North American and Greenland ground magnetometer arrays", Journal of Geophysical Research, 116 (A3), pp. 305, (2011). 

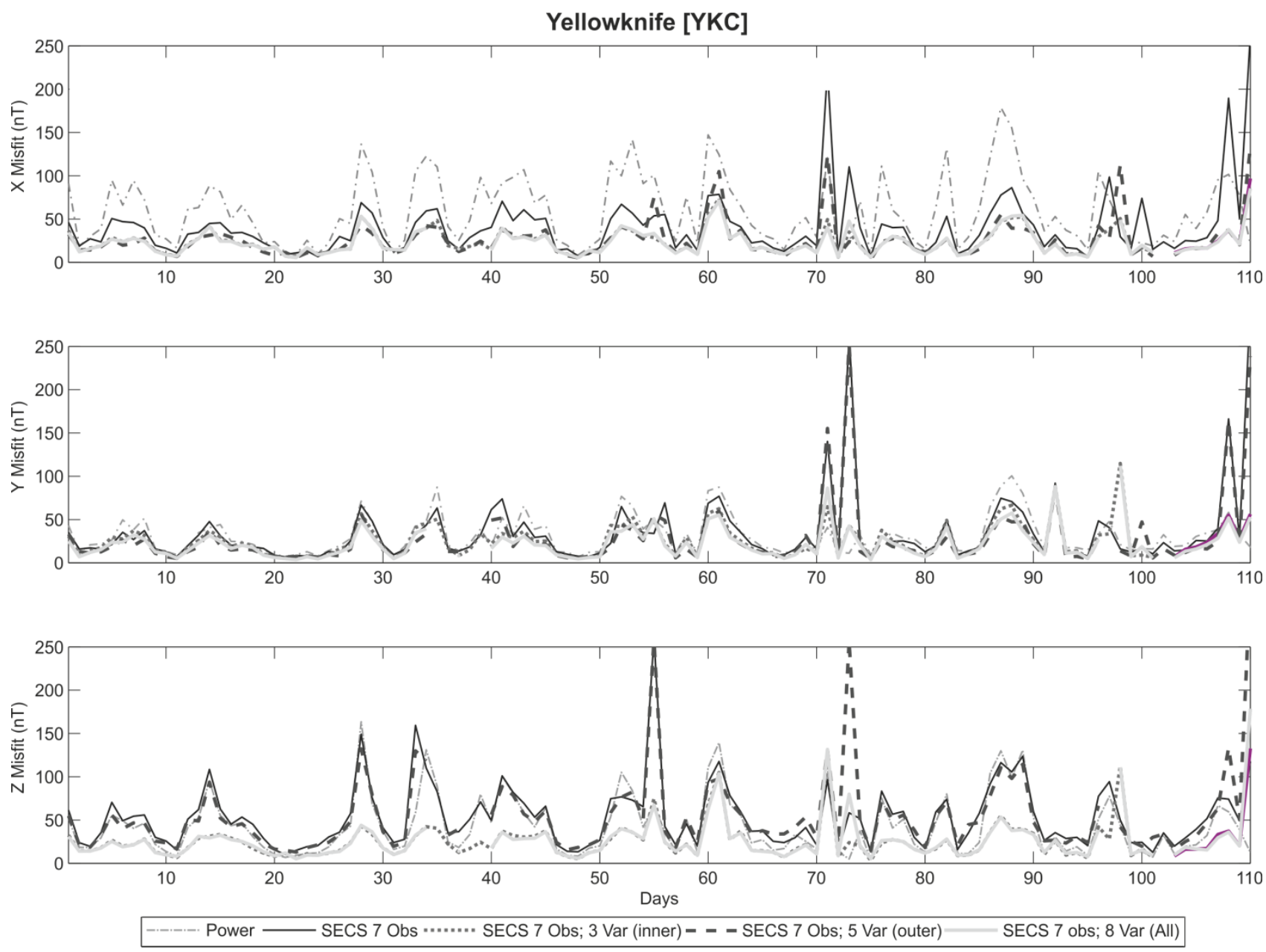

Figure 2: Comparison between Root Mean Square difference (in nanoTesla) of the daily power of measured external disturbances (gray dash) with the estimate from the SECS using seven observatories (black solid), SECS using seven observatories and the three closest variometers (grey dotted), SECS using seven observatories and the five distant variometers (black dash) and SECS using seven observatories and the all variometers (light grey solid) over a four month period (February-May 2007) at Yellowknife (YKC).

\begin{tabular}{|c|c|c|c|c|c|}
\hline \multicolumn{2}{|c|}{} & \multicolumn{4}{|c|}{ Mean SECS RMS Differences (nT) } \\
\hline & Power $(n T)$ & SECS 7 Obs & SECS 7 Obs; 3Var (inner) & SECS 7 Obs; 5Var (outer) & SECS 7 Obs; 8 Var (All) \\
\hline $\mathbf{X}$ & 59.1 & 41.2 & 23.5 & 34.9 & 22.9 \\
\hline $\mathbf{Y}$ & 31.4 & 33.0 & 25.3 & 27.2 & 22.3 \\
\hline $\mathbf{Z}$ & 42.9 & 56.0 & 25.5 & 51.6 & 26.3 \\
\hline
\end{tabular}

Table 1: Mean value of the daily power and of the Root Mean Square differences (in nanoTesla) of the measured external disturbances and the estimate from the SECS method with different numbers of observatories and variometers at Yellowknife (YKC). A difference lower than the Power indicates the SECS estimate is better than assuming no change occurs in the field. 